\title{
Non-parametric Diffeomorphic Image Registration with the Demons Algorithm
}

\author{
Tom Vercauteren ${ }^{1,2}$, Xavier Pennec ${ }^{1}$, Aymeric Perchant ${ }^{2}$, \\ and Nicholas Ayache ${ }^{1}$ \\ ${ }^{1}$ Asclepios Research Group, INRIA Sophia-Antipolis, France \\ ${ }^{2}$ Mauna Kea Technologies, 9 rue d'Enghien Paris, France
}

\begin{abstract}
We propose a non-parametric diffeomorphic image registration algorithm based on Thirion's demons algorithm. The demons algorithm can be seen as an optimization procedure on the entire space of displacement fields. The main idea of our algorithm is to adapt this procedure to a space of diffeomorphic transformations. In contrast to many diffeomorphic registration algorithms, our solution is computationally efficient since in practice it only replaces an addition of free form deformations by a few compositions. Our experiments show that in addition to being diffeomorphic, our algorithm provides results that are similar to the ones from the demons algorithm but with transformations that are much smoother and closer to the true ones in terms of Jacobians.
\end{abstract}

\section{Introduction}

With the development of computational anatomy and in the absence of a justified physical model of inter-subject variability, statistics on diffeomorphisms becomes an important topic [1]. Diffeomorphic registration algorithms are at the core of this research field since they often provide the input data. They usually rely on the computationally heavy solution of a partial differential equation [234] or use very small optimization steps [5]. In [6], the authors proposed a parametric approach by composing a set of constrained B-spline transformations. Since the composition of B-spline transformations cannot be expressed on a B-spline basis, the advantage of using a parametric approach is not clear in this case. In this work, we propose a non-parametric diffeomorphic image registration algorithm based on the demons algorithm. It has been shown in 7/8 that the original demons algorithm could be seen as an optimization procedure on the entire space of displacement fields. We build on this point of view in Section 2. The main idea of our algorithm is to adapt this optimization procedure to a space of diffeomorphic transformations. In Section 3, we show that a Lie group structure on diffeomorphic transformations that has recently been proposed in [1] can be used in combination with some optimization tools on Lie groups to derive our diffeomorphic image registration algorithm. Our approach is evaluated in Section 4 in both a simulated and a realistic registration setup. We show that in addition to being diffeomorphic, our algorithm provides results that are similar to the ones from the demons but with transformations that are much smoother and closer to the true ones in terms of Jacobians.

N. Ayache, S. Ourselin, A. Maeder (Eds.): MICCAI 2007, Part II, LNCS 4792, pp. 319-326, 2007.

(C) Springer-Verlag Berlin Heidelberg 2007 


\section{Non-parametric Image Registration}

\subsection{Image Registration Framework}

Given a fixed image $F($.$) and a moving image M($.$) , non-parametric image regis-$ tration is treated as an optimization problem that aims at finding the displacement of each pixel in order to get a reasonable alignment of the images. The transformation $s(),. p \mapsto s(p)$, models the spatial mapping of points from the fixed image space to the moving image space. The similarity criterion Sim (.,.) measures the resemblance of two images. In this paper we will only consider the mean squared error which forms the basis of intensity-based registration:

$$
\operatorname{Sim}(F, M \circ s)=\frac{1}{2}\|F-M \circ s\|^{2}=\frac{1}{2\left|\Omega_{P}\right|} \sum_{p \in \Omega_{P}}|F(p)-M(s(p))|^{2},
$$

where $\Omega_{P}$ is the region of overlap between $F$ and $M \circ s$. A simple optimization of (11) over a space of dense deformation fields leads to a ill-posed problem with unstable and non-smooth solutions. In order to avoid this and possibly add some a priori knowledge, a regularization term $\operatorname{Reg}(s)$ is introduced to get the global energy $E(s)=\sigma_{i}^{-2} \operatorname{Sim}(F, M \circ s)+\sigma_{T}^{-2} \operatorname{Reg}(s)$, where $\sigma_{i}$ accounts for the noise on the image intensity, and $\sigma_{T}$ controls the amount of regularization we need.

This energy indeed provides a well-posed framework but the mixing of the similarity and the regularization terms leads in general to computationally intensive optimization steps. On the other hand an efficient algorithm was proposed in 9 and has often been considered as somewhat ad hoc. The algorithm is inspired from the optical flow equations and the method alternates between computation of the forces and their regularization by a simple Gaussian smoothing.

In order to cast the demons algorithm to the minimization of a well-posed criterion, it was proposed in 7 to introduce a hidden variable in the registration process: correspondences. The idea is to consider the regularization criterion as a prior on the smoothness of the transformation $s$. Instead of requiring that point correspondences between image pixels (a vector field $c$ ) be exact realizations of the transformation, one allows some error at each image point. Considering a Gaussian noise on displacements, we end-up with the global energy:

$$
E(c, s)=\frac{1}{\sigma_{i}^{2}} \operatorname{Sim}(F, M \circ c)+\frac{1}{\sigma_{x}^{2}} \operatorname{dist}(s, c)^{2}+\frac{1}{\sigma_{T}^{2}} \operatorname{Reg}(s)
$$

where $\sigma_{x}$ accounts for a spatial uncertainty on the correspondences. We classically have dist $(s, c)=\|c-s\|$ and $\operatorname{Reg}(s)=\|\nabla s\|^{2}$ but the regularization can also be modified to handle fluid-like constraints $[7$.

\subsection{Demons Algorithm as an Alternate Optimization}

In order to register the fixed and moving images, we need to optimize (2) over a given space of spatial transformations. With the original demons algorithm, the optimization is performed over the entire space of displacement fields. These spatial transformations form a vector space and transformations can thus simply be added. This implies that we can use classical descent methods based on 
additive iterations of the form $s \leftarrow s+\boldsymbol{u}$. The interest of the auxiliary variable $c$ is that an alternate optimization over $c$ and $s$ decouples the complex minimization into simple and very efficient steps:

\section{Algorithm 1 (Demons Algorithm)}

- Choose a starting spatial transformation (a vector field) $s$

- Iterate until convergence:

- Given s, compute a correspondence update field $\boldsymbol{u}$ by minimizing $E_{s}^{\operatorname{corr}}(\boldsymbol{u})=\|F-M \circ(s+\boldsymbol{u})\|^{2}+\frac{\sigma_{i}^{2}}{\sigma_{x}^{2}}\|\boldsymbol{u}\|^{2}$ with respect to $\boldsymbol{u}$

- If a fluid-like regularization is used, let $\boldsymbol{u} \leftarrow K_{\text {fluid }} \star \boldsymbol{u}$. The convolution kernel will typically be Gaussian

- Let $c \leftarrow s+\boldsymbol{u}$

- If a diffusion-like regularization is used, let $s \leftarrow K_{\mathrm{diff}} \star c$ (else let $s \leftarrow c$ ). The convolution kernel will also typically be Gaussian

In this work, we focus on the first step of this alternate minimization and refer the reader to [7] for a detailed coverage of the regularization questions. By using classical Taylor expansions, we see that we only need to solve, at each pixel $p$, the following normal equations: $\left(J^{p T} . J^{p}+\sigma_{i}^{2}(p) \sigma_{x}^{-2} I\right) \cdot \boldsymbol{u}(p)=-(F(p)-$ $M \circ s(p)) . J^{p}$, where $J^{p}=-\nabla_{p}^{T}(M \circ s)$ with a standard Taylor expansion or $J^{p}=-\nabla_{p}^{T} F$ with Thirion's rule. From the Sherman-Morrison formula we get:

$$
\boldsymbol{u}(p)=-\frac{F(p)-M \circ s(p)}{\left\|J^{p}\right\|^{2}+\frac{\sigma_{i}^{2}(p)}{\sigma_{x}^{2}}} J^{p T}
$$

We see that if we use the local estimation $\sigma_{i}(p)=|F(p)-M \circ c(p)|$ of the image noise we end up with the expression of the demons algorithm. Note that $\sigma_{x}$ then controls the maximum step length: $\|\boldsymbol{u}(p)\| \leq \sigma_{x} / 2$.

\section{Diffeomorphic Image Registration}

In this section, we show that the alternate optimization scheme we presented can be used in combination with a Lie group structure on diffeomorphic transformations to adapt the demons algorithm.

\subsection{Newton Methods for Lie Groups}

Like most spatial transformation spaces used in medical imaging, diffeomorphisms do not form a vector space but only a Lie group. The most straightforward way to adapt the demons algorithm to make it diffeomorphic is to optimize (2) over a space of diffeomorphisms. We thus perform an optimization procedure on a Lie group such as in 1011. Optimization on Lie groups can often be related to constrained optimization by using an embedding. In this work we use an alternative strategy known as geometric optimization which uses the local canonical 
coordinates [11. This strategy intrinsically takes care of the geometric structure of the group and allows us to use unconstrained optimization routines.

Let $\mathcal{G}$ be a Lie group for the composition $\circ$. To any Lie group can be associated a Lie algebra $\mathfrak{g}$. $\mathcal{G}$ and $\mathfrak{g}$ are related through the group exponential which is a diffeomorphism from a neighborhood of 0 in $\mathfrak{g}$ to a neighborhood of Id in $\mathcal{G}$. The exponential map can be used to get the Taylor expansion of a smooth function $\varphi$ on $\mathcal{G}: \varphi(s \circ \exp (\boldsymbol{u}))=\varphi(s)+J_{s}^{\varphi} \cdot \boldsymbol{u}+O\left(\|\boldsymbol{u}\|^{2}\right)$, where $\left[J_{s}^{\varphi}\right]_{i}=\frac{\partial}{\partial u_{i}} \varphi(s \circ$ $\exp (\boldsymbol{u}))\left.\right|_{\boldsymbol{u}=0}$. This approximation is used in [11] to adapt the classical NewtonRaphson method by using an intrinsic update step:

$$
s \leftarrow s \circ \exp (\boldsymbol{u})
$$

One of the main advantages of this geometric optimization is that it has the same guaranteed convergence as the classical Newton methods on vector spaces.

\subsection{A Lie Group Structure on Diffeomorphisms}

The Newton methods for Lie groups are in theory really well fit for diffeomorphic image registration. In practice however it can only be used if a fast and tractable numerical scheme for the computation of the exponential is available. We would indeed have to use it at each iteration. Such an efficient scheme clearly relies on a good parameterization of the Lie group and the Lie algebra.

In the context of image registration, it has been proposed in [4] to parameterize diffeomorphic transformations using time-varying speed vector fields. This has the advantage of fully using the group structure. However the computation of a deformation field requires the numerical integration of a time-varying ODE. In [1] the authors proposed a practical approximation of such a Lie group structure on diffeomorphisms by using stationary speed vector fields only. This has the significant advantage of yielding very fast computations of exponentials. It becomes indeed possible to use the scaling and squaring method and compute the exponential with just a few compositions. On a strictly theoretical level, many technicalities arise when dealing with infinite dimensional Lie groups and further work is necessary to evaluate the well-posedness of this algorithm.

By generalizing to vector fields the equivalence that exists in the finitedimensional case between one-parameters subgroups and the exponential map, the exponential $\exp (\boldsymbol{u})$ of a smooth vector field $\boldsymbol{u}$ is defined in [1] as the flow at time one of the stationary ODE, $\partial p(t) / \partial t=\boldsymbol{u}(p(t))$. From the properties of one-parameters subgroups $(t \mapsto \exp (t \boldsymbol{u}))$, we see that for any integer $K$ we have $\exp (\boldsymbol{u})=\exp \left(K^{-1} \boldsymbol{u}\right)^{K}$. This yields the following efficient algorithm for the computation of vector fields exponentials:

\section{Algorithm 2 (Fast Computation of Vector Field Exponentials)}

- Choose $N$ such that $2^{-N} \boldsymbol{u}$ is close enough to 0 , e.g. $\max \left\|2^{-N} \boldsymbol{u}(p)\right\| \leq 0.5$

- Perform an explicit first order integration: $v(p) \leftarrow 2^{-N} \boldsymbol{u}(p)$ for all pixels

- Do $N$ (not $2^{N}$ !) recursive squarings of $v: v \leftarrow v \circ v$ 

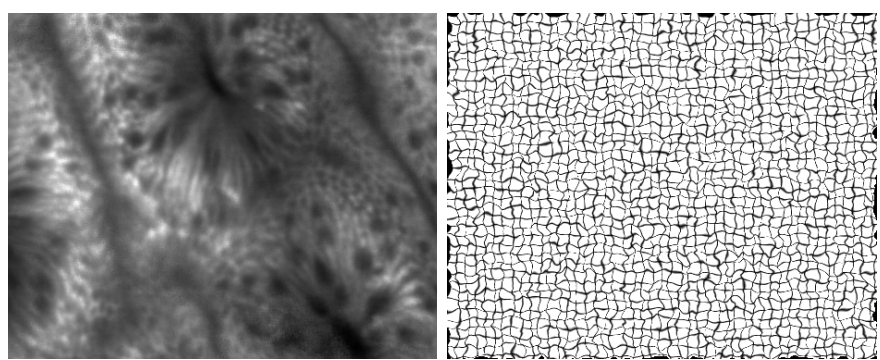

Fig. 1. Original image (FCM) of a normal human colonic mucosa image (image courtesy of PD. Dr. A. Meining, Klinikum rechts der Isar, Munich) and one example random warp used in our controlled experimental setup

\subsection{Efficient Diffeomorphic Demons}

We now have all the tools to derive our non-parametric diffeomorphic image registration algorithm. For the registration problem (2), the tools presented in Section 3.1 can be used to get the following approximation:

$$
F(p)-M \circ s \circ \exp (\boldsymbol{u})(p) \approx F(p)-M \circ s(p)+J^{p} \cdot \boldsymbol{u}(p)
$$

where $J^{p}=-\nabla_{p}^{T}(M \circ s)$ or $J^{p}=-\nabla_{p}^{T} F$ with Thirion's rule. Due to space constraints, we omit the technical details necessary to derive this approximation and refer the reader to 10 for a derivation on projective transformations.

As in Section 2.2. we focus on the first step of the minimization rather than on the regularization step. In order to get a computationally tractable expression of the correspondence energy, we chose the following distance between two diffeomorphisms: $\operatorname{dist}(s, c)=\left\|\mathrm{Id}-s^{-1} \circ c\right\|$. We then get $\operatorname{dist}(s, s \circ \exp (\boldsymbol{u}))=$ $\|\mathrm{Id}-\exp (\boldsymbol{u})\| \approx\|\boldsymbol{u}\|$. These approximations can be used to rewrite the correspondence energy used in the alternate optimization framework:

$$
E_{s}^{\mathrm{corr}}(\boldsymbol{u}) \approx \frac{1}{2\left|\Omega_{P}\right|} \sum_{p \in \Omega_{P}}\left\|\left[\begin{array}{c}
F(p)-M \circ s(p) \\
0
\end{array}\right]+\left[\begin{array}{c}
J^{p} \\
\frac{\sigma_{i}(p)}{\sigma_{x}} I
\end{array}\right] \cdot \boldsymbol{u}(p)\right\|^{2} .
$$

We see from (5) that we get the same expression as with the classical demons but that we consider $\boldsymbol{u}$ as a speed vector field instead of a deformation field. We thus obtain our non-parametric diffeomorphic image registration algorithm:

\section{Algorithm 3 (Diffeomorphic Demons Iteration)}

- Compute the correspondence update field $\boldsymbol{u}$ using (3)

- If a fluid-like regularization is used, let $\boldsymbol{u} \leftarrow K_{\text {fluid }} \star \boldsymbol{u}$.

- Let $c \leftarrow s \circ \exp (\boldsymbol{u})$, where $\exp (\boldsymbol{u})$ is computed using Algorithm 2

- If a diffusion-like regularization is used, let $s \leftarrow K_{\mathrm{diff}} \star c$ (else let $s \leftarrow c$ ). 

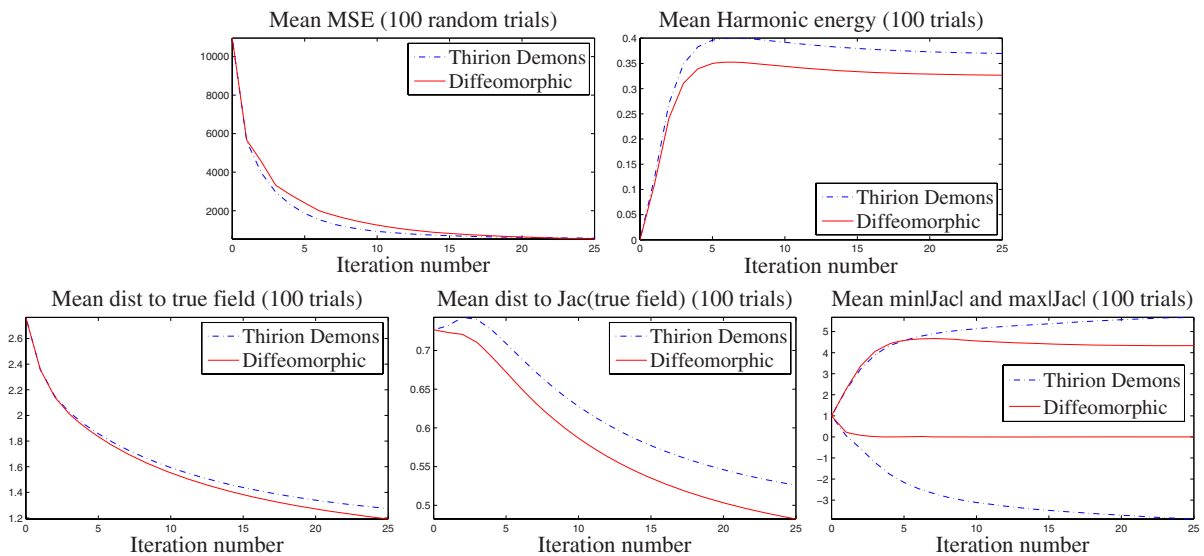

Fig. 2. Registration on 100 random experiments such as the one presented in Fig 1 Note that for similar performance in terms of MSE and distance to the true field, the diffeomorphic demons algorithm provides much smoother results and smaller distance to the true Jacobian of the transformation than the original demons algorithm. Most importantly we see that we provide diffeomorphic transformations whereas $\min (|\operatorname{Jac}(s)|)$ goes way below zero with the original demons algorithm.

\section{Registration Results}

To evaluate the performance of the diffeomorphic demons algorithm with respect to the original demons algorithm, two sets of results are presented. We used the same set of parameters for all the experiments: Thirion's rule with a maximum step length of 2 pixels was used in the demons force (3), a Gaussian fluid-like regularization with $\sigma_{\text {fluid }}=1$ and a Gaussian diffusion-like regularization with $\sigma_{\text {diff }}=1$ were used. Since the emphasis is on the comparison of the various schemes and not on the final performance, no multi-resolution scheme was used.

The first experiments provide a completely controlled setup. We use a fibered confocal microscopy image. For each experiment, we generate a random diffeomorphic deformation field (by passing a Markov random field through the exponential) and warp the original image. We add some noise both to the original and the warped image. We then run the registration algorithms starting with an identity spatial transformation. We can see on Fig. 2 that in terms of MSE and distance to the true field, the performance of Thirion's demons algorithm and of the diffeomorphic demons algorithm are similar. However the harmonic energy and the minimum and maximum values of the determinant of the Jacobian of the transformation show that our algorithm provides much smoother spatial transformations. We also see that our algorithm provides better results in terms of distance to the true Jacobian of the transformation. Moreover this is accomplished with a reasonable $50 \%$ increase of computation time per iteration with respect to the computationally efficient demons algorithm. 

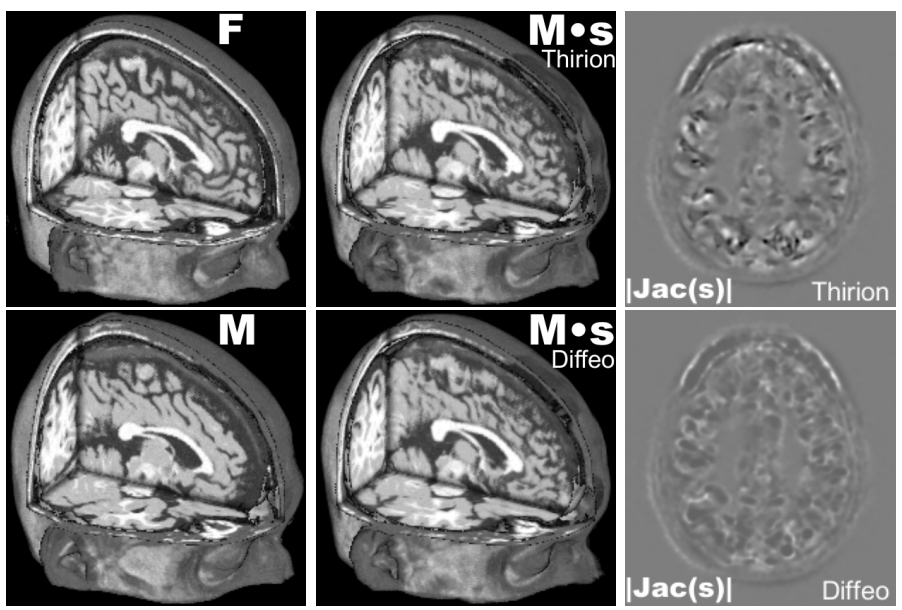

Fig. 3. Registration of two synthetic T1 MR images of distinct anatomies. For visually similar results, our algorithm provides smoother diffeomorphic transformations.

Our second setup is a more realistic case study were a gold standard is still available. We use synthetic T1 MR images from two different anatomies available from BrainWeb [12. These datasets are distributed along with a segmentation of eleven different tissue classes. We can see on Fig. 4 and Table 1] that on this dataset also, the demons algorithm and our algorithm provide very similar

Table 1. Comparison (Dice similarity coefficient $* 100$ ) of the discrete segmentations obtained from the registration of the synthetic T1-weighted MR images shown in Fig. 3

\begin{tabular}{|c||c|c|c|c|c|c|c|c|c|c|c|}
\hline & CSF & GM & WM & Fat & Muscle & Skin & Skull & Vessels & Fat2 & Dura & Marrow \\
\hline Initial & 41.73 & 63.06 & 61.51 & 19.30 & 20.14 & 66.65 & 42.75 & 14.26 & 6.06 & 14.74 & 28.19 \\
Thirion & 63.41 & 78.99 & 79.23 & 47.74 & 36.40 & 78.57 & 64.91 & 27.21 & 14.75 & 23.13 & 45.05 \\
Diffeo & 64.37 & 78.94 & 78.43 & 47.22 & 36.11 & 79.39 & 65.02 & 27.25 & 14.70 & 24.56 & 43.92 \\
\hline
\end{tabular}
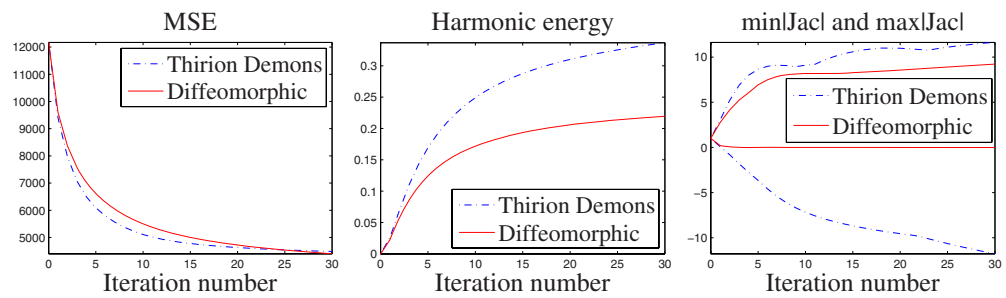

Fig. 4. Comparison of Thirion's demons algorithm with the diffeomorphic demons algorithm on the BrainWeb images shown in Fig. 3 . For similar performance in terms of MSE, our algorithm provides much smoother transformations than the original demons algorithm. Most importantly we see that we provide diffeomorphic transformations whereas $\min (|\operatorname{Jac}(s)|)$ goes way below zero with the original demons. 
results in terms of visual appearance, MSE and segmentation accuracy. However we see that our algorithm does it with much better spatial transformations. We indeed get smoother deformations that are diffeomorphic.

\section{Conclusion}

We have proposed an efficient non-parametric diffeomorphic registration algorithm. We first showed that the demons algorithm could be seen as an optimization procedure on the entire space of displacement fields. By combining a recently developed Lie group framework on diffeomorphisms and an optimization procedure for Lie groups, we showed that the framework in which we cast the demons algorithm could be adapted to provide non-parametric free-form diffeomorphic transformations. Our experiments have shown that our algorithm provides, with respect to the demons algorithm, very similar results in terms of MSE. This is however achieved with diffeomorphic transformations that are smoother and closer to the true transformations in terms of Jacobians.

\section{References}

1. Arsigny, V., Commowick, O., Pennec, X., Ayache, N.: A Log-Euclidean framework for statistics on diffeomorphisms. In: Larsen, R., Nielsen, M., Sporring, J. (eds.) MICCAI 2006. LNCS, vol. 4190, pp. 924-931. Springer, Heidelberg (2006)

2. Miller, M.I., Joshi, S.C., Christensen, G.E.: Large deformation fluid diffeomorphisms for landmark and image matching. In: Toga, A. (ed.) Brain Warping (1998)

3. Marsland, S., Twining, C.: Constructing diffeomorphic representations for the groupwise analysis of non-rigid registrations of medical images. IEEE Trans. Med. Imag. 23(8), 1006-1020 (2004)

4. Beg, M.F., Miller, M.I., Trouvé, A., Younes, L.: Computing large deformation metric mappings via geodesic flows of diffeomorphisms. Int'l. J. Comp. Vision 61(2) (February 2005)

5. Chefd'hotel, C., Hermosillo, G., Faugeras, O.: Flows of diffeomorphisms for multimodal image registration. In: Proc. ISBI 2002, pp. 753-756 (2002)

6. Rueckert, D., Aljabar, P., Heckemann, R.A., Hajnal, J.V., Hammers, A.: Diffeomorphic registration using B-splines. In: Larsen, R., Nielsen, M., Sporring, J. (eds.) MICCAI 2006. LNCS, vol. 4191, pp. 702-709. Springer, Heidelberg (2006)

7. Cachier, P., Bardinet, E., Dormont, D., Pennec, X., Ayache, N.: Iconic feature based nonrigid registration: The PASHA algorithm. CVIU — Special Issue on Nonrigid Registration 89(2-3), 272-298 (2003)

8. Modersitzki, J.: Numerical Methods for Image Registration. Oxford University Press, Oxford (2004)

9. Thirion, J.P.: Image matching as a diffusion process: An analogy with Maxwell's demons. Medical Image Analysis 2(3), 243-260 (1998)

10. Malis, E.: Improving vision-based control using efficient second-order minimization techniques. In: Proc. ICRA 2004 (April 2004)

11. Mahony, R., Manton, J.H.: The geometry of the Newton method on non-compact Lie-groups. J. Global Optim. 23(3), 309-327 (2002)

12. Aubert-Broche, B., Griffin, M., Pike, G.B., Evans, A.C., Collins, D.L.: Twenty new digital brain phantoms for creation of validation image data bases. IEEE Trans. Med. Imag. 25(11), 1410-1416 (2006) 